\title{
Scientific Evaluation of EMDR Psychotherapy for the Treatment of Psychological Trauma Summary: Scientific evaluation of EMDR psychotherapy
}

\author{
F. Haour ${ }^{*}$, E. Dobbelaere ${ }^{1,2}$, C. de Beaurepaire ${ }^{3}$ \\ 'EMDR France Association, 9 rue Papillon, 75009 Paris, France \\ ${ }^{2}$ Université Paris 5, 75006 Paris, France \\ ${ }^{3}$ EPS of Maison-Blanche, 6-10, Pierre-Bayle Street, 75020 Paris, France
}

Article Info

Article Notes

Received: December 14, 2018

Accepted: March 8, 2019

\section{${ }^{*}$ Correspondence:}

Dr. France Hour, EMDR France Association, 9 rue Papillon, 75009 Paris, France; Email: fhaour@gmail.com.

C 2019 Hour $F$. This article is distributed under the terms of the Creative Commons Attribution 4.0 International License
Summary

A recently described integrative psychotherapy, Eye Movement Desensitization and Reprocessing (EMDR), developed by F. Shapiro since 1989, has been confronted to the validation procedure used in pharmacological treatment (randomized control trials). This paper is a current review of the scientific validation steps of EMDR psychotherapy and its mechanisms of action. This EMDR therapy, focused on the resolutions of traumas, was started by treating patients with post-traumatic stress disorders (PTSD). The integrative EMDR protocol obtained the highest level of efficiency, for PTSD treatment. The efficiency of the protocol is now under study and scientific evaluation for troubles in which the trauma experiences are triggers or factors of maintenance of the troubles: anxiety, depression, phobia, sexual troubles, schizophrenia, etc. This new integrative psychotherapy follows the pathways and the timing observed for the evaluation and the validation of other therapies.

\section{Introduction: Assessment of Mental Disorders Treatments}

Biology, medicine, mental health and psychology have arrived late in the "scientific" field in the so-called "hard" sciences such as physics, chemistry and mathematics. Biology hbecame a "hard science" in the nineteenth century with Claude Bernard in $1865^{1}$. For mental disorders, this scientific approach appeared with the use of neuroactive substances in the middle of the last century, during the years 1950$1960^{2}$. For psychotherapies, the beginning can be seen in 1889 with the work of Pierre Janet ${ }^{3}$, but its development corresponds mainly to that of behavioral and cognitive therapies (CBT) by Ellis, Beck and Young ${ }^{4-7}$. These therapies have been scientifically evaluated very early because they followed experimental designs and control procedures that provide scientific evidence (Inserm randomized controlled trials ${ }^{8,9}$ ). In addition, the American Psychiatric Association (APA) ${ }^{10}$ published Diagnostic Manuals for Mental Health (DSM I in 1952, II in 1968, III in 1980, IV in 1994 and V in 2013) which have characterized psychic disorders. The same is true for the World Health Organization (WHO) ${ }^{11}$ : it proposed an International Classification of Diseases (ICD) from 1990 onwards. PostTraumatic Stress Disorder (PTSD), one of the main subjects of this article, was only characterized in 1980 by the DSM III and in 1992 by the ICD.

In 1989, a new structured therapeutic approach, the EMDR psychotherapy, was proposed for the treatment of trauma and PTSD. Over the last 25 years, this therapeutic approach has been submitted to the most demanding scientific assessment processes with respect to its clinical efficacy and mechanisms of action. 


\section{The Post Traumatic Stress Disorder (PTSD)}

The term "war neurosis" was used in the last two world conflicts to describe an anxious state with very various symptoms of repetition and avoidance. This state can last months and years, and is the consequence of one or more extremely traumatic events, such as exposure to combat episodes. The treatments were mainly focused on reducing the symptoms.

From 1980, the characterization and evaluation of the post-traumatic stress disorder (PTSD) and all the work identifying it to an anxiety disorder allowed to specify the symptoms ${ }^{10,11}$ : repetition, reviviscence of the event ("flashbacks" and nightmares), sleep disorders, irritability, hypervigilance, avoidance of experiences linked to trauma, detachment, restriction of affects. PTSD is called "acute" if the duration of symptoms is less than three months, and "chronic" beyond. This diagnosis has led to a better understanding of the mental mechanisms involved, an assessment of post-traumatic stress disorder and the development of trauma-focused psychotherapeutic approaches, alongside pharmacological treatments ${ }^{2,12,13}$.

\section{The beginning of EMDR psychotherapy}

Claude Bernard (1865) observed at the end of the nineteenth century that "it is always by chance that everything begins", that "science comes only after" and that "its reasons on what chance has shown". True for somatic medicine, this observation is also true for psychiatry: fortuitous efficacy of a therapy on a symptom, proof of this effectiveness through the practice of controlled and randomized trials, followed by research on the mechanisms of action.

The history of EMDR begins with an individual observation of Francine Shapiro in California. This young American student was very interested in the interactions between body, brain and mental states. In addition, she was experiencing a personal problem, a well-treated cancer but nevertheless a source of concern for her own health. She was finally at a time in the history of psychology, the 1970s-80s, in a region of the world where new approaches emerged : brief therapies (Palo Alto, School, Mental Research Institute), psycho-neuro-immunology, study of the role of emotions on health with Norman Cousins and development of cognitive-behavioral therapies. It was also the time of the Vietnam war, when soldiers returned traumatized, in a state of post-traumatic stress, unable to resume a normal life and without efficient therapy to cure them.

In this context, it is by chance that she observed that her alternated eye movements (left / right), improved her mental state. She observed the same effect by consciously repeating the experience on herself. In this favorable sociohistorical context, she tested the procedure involving eye movements on veterans from Vietnam who presented the clinical criteria for a post-traumatic stress disorder (PTSD) and then, thanks to her training, she developed the eclectic EMDR protocol. She introduced Joseph Wolpe's "Subjective Unit of Disturbance" scale (SUDs) ${ }^{14}$, who used desensitization techniques to treat phobias and anxietyprovoking stimuli. Two articles related to her initial clinical work were published in $1989^{15,16}$. They showed that the treatment was rapidly effective and appeared to lead, in PTSD, to desensitization of anxiety symptoms related to traumatic memories. It therefore allowed treatment of PTSD recently included in the DSM III.

The scientific community has long been skeptical ${ }^{17}$, but Francine Shapiro thanks to her energy, conviction, personal experience and multiple skills, succeeded in creating an EMDR Institute. The protocol then was taught to a large number of psychotherapists ${ }^{18,19}$ who practiced EMDR with rapid and positive clinical results that led Francine Shapiro to hypothesize the Adaptive Information Processing (AIP) ${ }^{20,21}$. According to this theoretical model, under normal conditions, trauma resolution is spontaneous. It does not seem possible when the brain has been "overwhelmed" by too intense or repeated trauma. The memory of the different components (emotional, sensory, cognitive and physiological) of the trauma are then stored in a "dysfunctional" way, unprocessed. Eye movements seem to favor the restarting of the processing of dysfunctionally stored pieces of information, as well as their desensitization and integration.

The term "dysfunctional" refers to maladaptation to current situations. The recall of the trauma and the existence of related symptoms induce responses that are no longer justified by the current situation. This hypothesis comes from research on the neurobiological mechanisms of stress and trauma as well as the mechanisms of different types of memory. The mechanisms of memory, recall of memories and their reconsolidation are also here a very studied and important process ${ }^{22,23}$. The AIP hypothesis is consistent with the analysis of clinical practice that shows dramatic and rapid changes in emotional state and vividness of traumatic memories during a 90-minutes EMDR psychotherapy session.

The treatment, including eye movement sequences, has become "Eye Movement Desensitization and Reprocessing : EMDR "with an eight-phase protocol (in the Appendix) that targets and treats the traumatic events of the past, their consequences in the present, and anxiety linked to future events ${ }^{20,21}$. The protocol integrates in a structured way various active elements from different psychotherapeutic approaches (empathic listening, psycho-education, psychometric tests, cognitive restructuring, association of memories, modification of inappropriate early schemas, use of Socratic interrogation and metaphors, exhibition 
in imagination, desensitization, relaxation, work taking into account sensations, behavior and systemic context, mindfulness ...). It is therefore an "integrative" psychotherapeutic approach.

The World Health Organization's 2013 report ${ }^{22}$, recommends two therapeutic approaches for the treatment of PTSD: CBT and EMDR. It underlines the differences between these two psychotherapies: "Like CBT with a trauma focus, EMDR aims to reduce subjective distress and strengthen adaptive beliefs related to the traumatic event. Unlike CBT with a trauma focus, EMDR does not involve (a) detailed descriptions of the event, (b) direct challenging of beliefs, (c) extended exposure, or (d) homework." According to Swift and Greenberg's meta- analysis ${ }^{23}$, "integrative treatments result in the lowest dropout rates for depression and PTSD". In contrast, the highest scores of premature terminations in PTSD are observed in various CBT modalities using a strong exposure component. However, Ho \& Lee 2012' s meta - analysis ${ }^{24}$ revealed "a difference in the prescribed homework" between these two treatments of PTSD: EMDR requires much less hours of homework than CBT. Then the main differences between CBT and EMDR and CBT seems to be the degree of exposure to trauma and the amount of homework.

\section{The Structure and Stages of the Protocol (8 Phases)}

Sessions last from $50^{25}$ to 90 minutes, the protocol includes 8 phases. Phases 1 and 2, common to other psychotherapeutic approaches (in particular cognitive and behavioral therapies [CBT]), the patient and the clinician establish a "targeting plan" of the trauma (s) to treat. The treatment of an isolated trauma is achieved in one or in/ a few sessions, while childhood trauma or repeated trauma requires more sessions. Phases 1 and 2 of the protocol all available approaches in psychotherapy can be usedcognitive-behavioral, body therapy, hypnosis, relaxation, psychodynamic therapy - in a very strict and reassuring framework for the patient.

Phase 3 is the quantitative phase that assesses the mental state of the patient at the beginning of the traumatic target reprocessing session.

Phases 4 to 8 are EMDR specific and are based on the assumption of adaptive information processing (AIP). During an acute or repeated traumatic event, this process is sometimes not available and the memory of the trauma cannot be "integrated". It remains "active" in the patient's current behavior as observed in post-traumatic stress disorder. To treat this or these traumatic events, the patient must remind it.

For each session, from a specific "target" event, the patient is guided and supported towards the traumatic scene that he "retreats" spontaneously, in the safety of the therapeutic context, using bilateral alternated stimulations. (BAS). At the end of each set of BAS, the patient verbalizes his emotions and perceptions. The alternated BAS and verbalizations favor the processing of emotions and memory in the presence of an empathic and reassuring professional. It is the desensitization phase, in which the traumatic memory loses its "emotional" tone and its precision. Bilateral alternated stimulations promote and accelerate this process of desensitization. The patient realizes that the emotions, beliefs and sensations associated to the traumatic event were appropriate at the time of the traumas (fear, shame, guilt, sadness) but no longer in the present moment. This process happens during a session, while the evolution of the mental state of the patient is evaluated periodically through the VOC and SUD scales as indicated in Appendix 1 which describes the protocol in detail. The traumatic target is recalled several times in the same session and is treated in the next session if the evaluation of desensitization shows that it is not complete. Work is also being done on the present through work on "current triggers" and on the future, i.e. on "anticipations". During the reprocessing phases (3 to 8), the therapist is asked to intervene as little as possible so that the recall and re-encoding work is done in a free and spontaneous way. The reprocessing speed can be very fast for isolated traumas but requires multiple sessions in the case of repeated or ancient traumas.

This highly structured and "synclectic" approach ${ }^{20-21,}$ with continuous monitoring of the patient's mental state, ensures fast and stable therapeutic results. The effects obtained are maintained as shown by tests carried out after several months and are definitive for isolated traumas.

The protocol, during reprocessing, focuses on three existential themes: responsibility (shame and guilt), safety (fear, vulnerability) and the possibility of choice (control, self-esteem) ${ }^{19-21}$.

(for a more detailed description of the protocol, cf. annex 2).

\section{Scientific assessment of EMDR Psychotherapy}

\section{Evaluation of EMDR therapeutic effect in PTSD}

a) The first scientific verification of the EMDR protocol effectiveness in the treatment of pathologies related

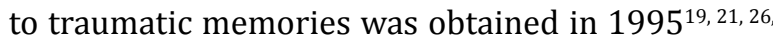
27.

b) Controlled and randomized clinical studies show that EMDR is efficient to treat PTSD ${ }^{28-33}$ and that it gives very significant results in comparison to the absence of treatment or to other pharmacological or psychotherapeutic approaches ${ }^{34}$.

It turns out to be very useful to treat refugees and victims of disasters experiencing PTSD ${ }^{35-37}$. 
Different ways to use EMDR are efficient to treat PTSD: intensive treatments ${ }^{38}$, group treatment ${ }^{39,40}$.

EMDR therapy has also been used successfully for the treatment of traumatized children. Randomized controlled studies and a meta-analysis ${ }^{41-44}$ demonstrate its effectiveness in treating trauma in children.

Furthermore, it's efficient to prevent PTSD with early interventions after traumas ${ }^{45,46,47}$.

c) Several meta-analyzes ${ }^{20,48-51}$ demonstrate the clinical efficacy of the EMDR protocol for PTSD. This research and evaluation has made it possible to compare its effects favorably to those obtained with CBT (trauma-focused TBIs) and pharmacological treatments. Bilateral Alternating stimulations are most often obtained by eye movements. More recently, auditive and tactile stimulations have also been used. There are currently more than 750 published articles on EMDR regarding clinical outcomes and mechanisms of action. Some articles synthetically review this literature ${ }^{53,54}$.

\section{Evaluation of EMDR therapeutic effect in various mental disorders}

The most recent publications also show the effectiveness of EMDR in the treatment of various mental disorders: acute stress $^{55}$, depression ${ }^{56}$, phobias $^{57-60}$, addictions ${ }^{61-63}$, chronic pain $^{64,65}$, Obsessive- compulsive disorder ${ }^{66,67}$, self-esteem ${ }^{68}$, autism $^{69}$ and schizophrenia ${ }^{70}$. In the case of patients treated for schizophrenia, EMDR therapy was used because many of them had symptoms of post-traumatic stress ${ }^{70-75}$. On the other hand, there is a correlation between the importance of childhood trauma and the probability of developing a state of schizophrenia.

High levels of evidence are observed in these articles but often, they do not yet reach the maximum level achieved in randomized controlled trials. Therefore, an EMDR treatment targeting all traumatic life events, may have therapeutic effects in various disorders and pathologies.

\section{Recommendation by health organizations}

EMDR therapy is now recognized as a post-traumatic stress disorder therapy in different countries (England, United States, Holland, France ...) in the context of "evidence-based medicine"10-13. In July 2013, the World Health Organization (WHO) published recommendations and guidelines ${ }^{22}$ for practitioners dealing with the consequences of acute traumas (violence or views, accidents, war, bereavement) leading to post-traumatic stress disorder (PTSD) in adults, children and adolescents. The French High Authority for Health (HAS) also recommends the use of EMDR psychotherapy ${ }^{12}$. The WHO showed that $3.6 \%$ of the world population has suffered from PTSD. The validation of the efficacy of a treatment of this disorder is therefore particularly important.

\section{Mechanisms of action}

\section{Role of Bilateral Alternating Stimulations}

Several neurophysiological mechanisms have been considered to explain the effect of bilateral alternated eye stimulations: analogy with eye movements observed in REM sleep, changes in ortho-parasympathetic equilibrium, facilitation of inter-hemispheric interactions, double attention, distraction (taxing the working memory), orienting reflex.$^{76-77}$. The most recent results emphasize the role of eye movements in recalling the memories stored in different parts of the brain dealing with memories, in particular in the working memory. This memory has a limited capacity and when two tasks, recalling a memory and eye movements, are requested at the same time, this memory is taxed and the memory becomes more vague and less emotional ${ }^{77-82}$. In general, each recall of a memory is likely to modify it and it is returned and reconsolidated in the long-term memory in this modified form. Recent work $^{22,23}$ on the mechanisms of memorizing, recalling and re-encoding of memories give a neurobiological basis to these hypotheses.

If the distraction hypothesis is correct, any procedure that produces double attention or "distraction" during memory recall will reduce the precision and the emotional impact of traumatic memories. In experiments carried out on control adults, it is observed that mental calculation, the game "Tetris" and the concentration on one's breathing ${ }^{81,82}$ confirm this effect. This latter hypothesis could provide an explanation for the efficacy of alternated bilateral auditory or sensory (alternating pattern) stimulation in the process of desensitization.

However, the different types of "distraction" do not have the same degree of relative effectiveness. Indeed, it appears that alternate ocular stimuli are more effective than other types of stimulation ${ }^{78,79,83}$.

Some researchers hypothesized the implication of slow waves sleep in EMDR psychotherapy ${ }^{84,85}$ and focus on the similarity between the "EMDR Eye Movements and delta and beta waves occurring during the Slow Wave Sleep. SWS has a key role in memory consolidation and in the reorganization of distant functional networks, as well as Eye Movements seem to lead to a weakening of traumatic episodic memory and a reconsolidation of new associated information".

Finally, brain imaging showed changes thanks to the EMDR treatment in PTSD patients. Recordings made during an EMDR session have specified the areas involved 
preferentially, limbic, parietal and temporo-occipital cortex $^{86-88}$.

In addition, different changes in the brain were shown after EMDR treatment: grey matter density changes ${ }^{89}$, changes in the brain connectivity ${ }^{90}$, change in the amygdala volume $^{91}$.

The hypotheses suggested by these observations must naturally be validated in order to specify the mechanisms at work.

More recently, Khalaf \& al.'s research ${ }^{92}$ suggests that the reactivation of the original memory of fear actively contributes to remote fear attenuation" and points out the activation of memory recall-induced neurons. Furthermore, de Voogd \& al. ${ }^{93}$ showed that eye movement interventions enhance extinction thanks to the deactivation of the amygdala, as does exposition.

\section{Role of the orientating response and Rapid Eye Movements}

\section{The orientating response}

Many neurobiological studies and a number of theoretical models consider the notion of orientating response or orientating reflex (Pavlov, 1927, Sokolov, 1963) as one of the mechanisms of action of EMDR. According to the orientating response theory, bilateral eye movements (or alternating bilateral stimulations) activate an "investigative reflex" which is first manifested by an alert response, followed by a pause that produces a deactivation in front of an absence of threat.

The orientation response is a physiological process that directs attention towards a new and meaningful stimulus. The terms "orientating reflex" or "orientating reaction" refer to the mobilization of attention following a change in the environment. This response orientates towards the processing of information by comparing new information with familiar, known information.

According to some researchers, EMDR could generate an orientating response that suppresses emotional disturbances. Denny ${ }^{94}$ proposed an inhibition model in which an orientating response suppresses the emotional disturbance associated with traumatic memory. This relaxation response acts to desensitize a traumatic memory or episode lived negatively. It is physiologically incompatible with the anxiety that emerges when one thinks of a non- treated traumatic memory.

\section{REM}

According to Stickgold ${ }^{95,96}$, the orientating response model states that eye movements performed in EMDR produce a brain state similar to that of REM sleep, reactivating the mechanisms of REM sleep. REM sleep serves adaptive functions, and in particular memory consolidation. Our brain uses eye movements, at night, to integrate the information recorded during the day. Stick gold recalls that, during the paradoxical sleep phase, during the dream, weakly associative material is transferred, thanks to acetyl cholinergic mechanisms, from the neocortex to the hippocampus (involved in the formation of long-term memory). On the contrary, during the other phases of sleep, strongly associative material of the hippocampus is transferred to the neocortex. This double transfer weakens the emotional charge of memories.

Post-traumatic stress is accompanied by a disruption of sleep and the acetyl cholinergic mechanism, which diminishes the possibility of weakening these strong associations and reducing the emotional impact of the trauma. The use of EMDR could generate an increased production of acetylcholine, which could stimulate the transmission of information from the cortex to the hippocampus. The alternating bilateral stimulations could favor a decoding of information, memories, similar to the decoding taking place during paradoxical sleep.

Noticing the parallels between REM sleep and EMDR, Stick gold argued that EMDR reduces the symptoms of posttraumatic stress disorder by transforming emotionally charged traumatic memories into semantic memories. This author suggests that the constant reorientation of attention caused by bilateral stimulations (visual, auditory or tactile) produces a sufficient load of acetylcholine and an orientating response, consequently facilitating the activation of a cerebral state similar to REM sleep.

\section{Role of the structure and the steps of the EMDR protocol}

A scientific evaluation of all the protocol steps is not easy. This protocol uses a set of psychotherapeutic approaches and involves emotions, cognitions and body sensations, while giving great importance to the assessment of the patient's mental state by himself. This structure, however, creates a state of safety for the patient and the therapist, one of the causes, no doubt, of the therapeutic action. Furthermore, the empathetic listening ability of psychotherapists facilitate the change processes.

\section{Discussion and Conclusion}

It seems that EMDR psychotherapy is in the classic situation of important advances and discoveries: a period and a favorable environment, an observation and an original idea, a strong personality and the availability of scientific infrastructures to verify a hypothesis and its effects. Establishing the scientific proof of a therapeutic action is a long process involving many actors: researchers and practitioners, hospitals, publications, statisticians, metaanalyzes, evaluations and recommendations by national and international bodies. This process usually takes more than 20 years so that the effectiveness of a treatment is recognized 
well before one understands its mechanisms. From this viewpoint, EMDR psychotherapy is in a development stage similar to that experienced by other therapies.

EMDR treatment was initially used to treat PTSD because it allows desensitization of emotions, thoughts and physical sensations experienced during traumatic experiences. In addition, its protocol was shown to be effective for the treatment of pathologies (anxiety disorders, depression, schizophrenia, etc.) usually treated with a various pharmacological or psychotherapeutic approaches. It is has become clear thatEMDR treatment is useful in the treatment traumatic events, triggers or aggravating factors of a large number of psychiatric disorders. EMDR psychotherapy has benefited from the extremely important work done in the field of psychopathology and neurobiology and presents itself as an integrative and "synclectic" therapy. In the most serious cases, it can be performed in co-therapy with an appropriate pharmacological treatment (depression, generalized anxiety, schizophrenia). This synergy between pharmacological treatment and EMDR psychotherapy represents a complementary and promising approach for the treatment of psychopathological disorders.

\section{Declaration of interest}

France Haour, Emmanuelle Dobbelaere and Christiane de Beaurepaire certify that they have no conflict of interest regarding the scientific evaluation of EMDR psychotherapy.

\section{To know more}

\section{Annex 1: EMDR protocol}

The EMDR protocol, focused on the resolution of painful and traumatic events (memories), has 8 phases. Bilateral Alternated Stimulations (BAS) occur in phases 4 to 7 [20-22]. 1-Patient's history : Current problem, conceptualization of the case, traumatic targets ; 2 - Preparation : psychoeducation, "safe" place, stress management. 3 - Evaluation : traumatic memory target, Negative and positive cognition, VOC (validity of cognition : Scale 1-7), emotions (fear, sadness, shame, disgust, guilt), SUDs (Subjective Unit of Disturbance scale : 0-10), body sensations. 4 - Desensitization (and adaptive reprocessing) : sensory image, cognition, body sensations ; BAS series ; re-evaluation of SUDs and VOC. 5 - Installation of the positive cognition (reprocessing) ; series of BAS. 6 - Body scan (reprocessing) ; BAS. 7 - Safe closure. 8-Reassessment at the next session. Return to the initial target and check that all links with present, past and future have been reprocessed. If not, a new session is scheduled.

The standard EMDR protocol, as described below, consists of 8 phases :

Phase 1 is the history taking (: Current problem, conceptualization of the case, traumatic targets).
In Phase 2 (preparation), the EMDR process is explained and the "safe place", a guided visualization in a place where the participant feels good and / or safe, is installed. In normal use of standard EMDR, this phase can be expanded, as it can include a long development and installation of resources, and self-regulation techniques, such as in a wide range of therapies or coaching.

Phase 3 is dedicated to identifying and evaluating a distressing event or target. The client is asked to evaluate the distress caused by the event, using the unit of subjective disturbance units (SUD) (Wolpe, 1969). In addition, the validity of a preferred positive self-referential cognition is assessed, related to the situation, using the validity of the cognition scale (VOC, Shapiro, F. 2001, Shapiro, F., 1989).

In phase 4 (desensitization), 5 (installation of positive cognition) and 6 (body scan), the disturbing material is processed, using double attention: the client focuses on the target and simultaneously on an external visual stimulus, follows the movements of the therapist's hand by performing eye movements. Reprocessing a trauma may require one to several therapy sessions.

To desensitize (phase 4) a traumatic episode, or a painful memory, or a future episode that the client apprehends, the psychotherapist invites the patient to focus on the image of this event, by being in contact with its sensory elements (visual, auditory, olfactory, kinesthetic, even taste), with the beliefs and negative emotions that he has generated. The therapist then initiates series of alternating (left and right) bilateral stimulations : eye movements, sounds or tactile stimulations. As time goes by, associations of ideas (free associations, in a Freudian language) emerge and patients retreat the various information related to the memory, integrate them into their memory network in a functional, adapted way (episodes "pass " from the amygdala to the pre-frontal cortex). Between each series, the therapist asks the patient to take a deep breath and share what came to mind during stimulation.

In phase 5 , which occurs after the desensitization of negative emotion, the desensitized memory is associated with positive cognition (what the client would like to think of him in relation to this context, for example: "I can cope with "," I am safe, "' I am a good person "), and while he is thinking about this cognition as well as what is left of the representation of the reprocessed situation, other series of alternating bilateral stimulations are performed. When the patient can rethink the memory without emotional embarrassment and associate it with positive thoughts (phase 5 and 6), the bilateral stimulations are stopped.

In phase 6 , the client scans his body by closing his eyes and observing if there are any emotional disturbances or physical tensions. If so, another desensitization is applied to relieve them. At the end of treatment, clients generally 
observe that distress has disappeared, positive cognition is stronger, if not totally true, and all related physical disturbances have been eliminated. The alternating bilateral stimulations thus desensitized the memory of the trauma and reprogrammed the negative beliefs related to it.

Phase 7 is the closure of the session.

Phase 8 is a reassessment of previous work, normally done at the next session.

\section{Annex 2 Transcript of an EMDR session}

This transcript can be found in the Permanente Journal / Winter 2014/ Volume 18 No. 1

"Partial Transcript of Eye Movement Desensitization and Reprocessing Therapy Session1 A history-taking session revealed that "Lynne" had experienced 2 earthquakes. The first had occurred while in a college hypnosis class. The professor had just put her under hypnosis when the earthquake struck. However, she did not report any PTSD symptoms until years later, after the second earthquake when she was alone in the house with her son. After a preparation phase that teaches techniques to ensure adequate emotional stability,1,2 the second earthquake was targeted. Processing begins after suitable preparation and assessment. The latter includes identification of a) the worst part of the event (cowering in the doorway with her son), b) the negative belief (I'm powerless), c) a desired positive belief (I can handle whatever comes up), d) the current emotional response (anxiety), and e) body reaction (feeling in stomach), as well as the measurement of negative affect ( 8 on an 11-point scale) and strength of the desired positive belief ( 2 on a 7 -point scale). After initial concentration on an image of cowering in the doorway with her son, the negative belief "I'm powerless," and the negative feeling in her stomach, the following associations emerge with each set of eye movements. During each set, Lynne is instructed to simply "Notice whatever comes to mind, and let whatever happens, happen." At the end of each set, Lynne is told to "Blank it out" or "Let it go, and take a deep breath." After that, she is asked some version of, "What are you noticing now?" After she reports, the clinician guides the next focus of attention according to standardized protocols. In this excerpt, Lynne is generally directed to think about what has just emerged in consciousness. The designation >>>>> means that her eyes are being guided in a set of eye movements. It is during that time that the associations and connections are made. She then reports them to the clinician. Note that at times a seemingly innocuous event is a precursor to recalling an experience that is disturbing and related to feelings of powerlessness. >> >> Lynne: Ah. Sort of a softness in my body. I'm ... more aware ... my legs feel really heavy. Sort of a sinking kind of feeling in my legs - they just feel kind of woozy. >>>>> Lynne: The first thing that occurred to me was just the tape that I listened to about the people who work on trains and-and have to watch people get smashed by trains. That's the first thing that occurred to me. Then I just started being more aware of my body again. Not having another thought. >>>>> Lynne: More tiredness in my upper body. Sad, melancholy feeling. >>>>> Lynne: I just flashed on the earthquake happening where I was in the hypnosis class and, and I was under and the earthquake happened. >>>>> Lynne: Just feeling really tired. Noticing my body, really tired. >>>>> Lynne: (laughing) I was thinking about running around the house with my brother when I was six. I wanted to be a boy, and he told me if I ran around the house enough times, I would be a boy. And I was disappointed because it didn't happen. >>>>> Lynne: Yeah I was thinking about my sense of betrayal with my brother that he molested me, and how I really admired him (crying). >>>>> Lynne: Yeah. (crying) Something occurred to me like "Duh": How much that shook my sense of reality. >>>> Lynne: I was thinking about playing cards with my dad across the table from one another. $>>>>>$ Lynne: I was thinking about my dad taking me to buy a coat and buttoning the button of my coat pinching my nipple when I was like eleven and how absolutely stunned I was with that. >>>> Lynne: What comes really clear is getting sick when I was around the same age. Getting really sick with a pain in my side and nobody being able to figure out what it was and being rushed to the hospital. I really couldn't lower my leg, and no one could decide what was wrong with me. I was experiencing a really bad pain in my side and then they just decided that I had some kind of mental problem. I guess that was the only way that I could express it. >>>>> Lynne: Gosh, I was just thinking what a chaotic place it was to live in and what an unsafe place it was to be. >>>>> Lynne: I was thinking of my mom and dad fighting and throwing things at one another while we were supposed to be in bed asleep and hiding under the bed and trying to go to sleep and being afraid. >>>> Lynne: I was thinking about how I wanted to protect my dad from my mother. It seemed really crazy. >>>> Lynne: It kind of came back up to the earthquake in ' 87 and jumping out of the shower, and running in and gabbing my son out of the crib and running with him downstairs and trying to protect him. Note how a feeling of power is emerging. After a few more sets Lynne is asked to return to the original incident : Lynne: I can see it, truly I can see it, but it doesn't have-doesn't really have a feeling component right this second. Clinician: Okay. And that desired positive statement: "I can handle whatever comes up"? Lynne: That sounds fine. Yeah, I can handle whatever comes up. As indicated in Table 1, further processing is used to strengthen the positive belief and eliminate any residual physical disturbance. In addition, instructions are given to ensure between-session stability and adequate reevaluation. In further sessions, current 
trigger and future needs would be addressed. Detailed descriptions of clinical sessions are available in various publications."

\section{Annex 3: References}

1. Bernard C. Introduction à l'étude de la médecine expérimentale. Paris Champs Flammarion. 2008. 1865.

2. Stahl SM. Stahl's Essential Psychopharmacology, Neuroscientific basis and practical applications. 1st ed. Cambridge : Cambridge University Press. 2008.

3. Janet P. Études expérimentales sur les troubles de la volonté, de l'attention, de la mémoire sur les émotions, les idées obsédantes et leur traitement. In : Névrose et idées fixes. Paris Masson. 1998.

4. Ellis A. A cognitive approach to behavior therapy. Int J Psychiatry. 1969; 8(6): 896-900.

5. Clark DA, Beck AT. Cognitive therapy of anxiety disorders : scienceandpractice. Guilford Press. 2010.

6. Young JE, Klosko JS, Weishaar ME. La thérapie des schémas : approche cognitive des troubles de la personnalité. Bruxelles : De Boeck. 2005; 2003.

7. Cottraux J. Les thérapies comportementales et cognitives. Paris Masson Éd. 2004.

8. Inserm Psychothérapies Trois approches évaluées. Une expertise collective de l'Institut national de la santé et de la recherche médicale. Inserm. 2004.

9. Thurin JM, Thurin M. Évaluer les psychothérapies méthodes et pratiques. Paris Dunod. 2007.

10. Diagnostic and statistical manual of mental disorders. 4th ed Washington DC : American Psychiatric Association ; 2000.

11. International Classification of Deseases. (2018). WHO. https:// icd.who.int/browse11/l-m/en\#/http://id.who.int/icd/ entity/2070699808

12. Haute Autorité de santé (HAS). Troubles anxieux graves. 93218SaintDenis-la- Plaine. 2007.

13. NICE (The National Guidelines of Health and Clinical Excellence) Post-Traumatic stress Disorders (PTSD) : The management of PTSD in adults and children in primary and secondary care 2005, Guidelines [CG26] ; 2005.

14. Wolpe J. The practice of behaviour therapy. Oxford : Pergamon Press. 1969.

15. Shapiro F. Efficacy of the eye movement desensitization procedure in the treatment of traumatic memories. J Traumatic Stress. 1989; 2 199-223.

16. Shapiro F. Eye movement desensitization : a new treatment for posttraumatic stress disorder. J Behav Ther Exp Psychiatry. 1989; 20 211-7.

17. Muris P, Merckelbach H. Traumatic memories, eye movements, phobia, and panic : a critical note on the proliferation of EMDR. J Anxiety Dis. 1999; 13: 209-23.

18. Maxfield L. EMDR milestones : the first 20years. J EMDR Pract Res. 2009; 3: 211-6.

19. Shapiro F. Eye movement desensitization and reprocessing : basic principles, protocols and procedures. New York : The Guilford Press. 1995.

20. Shapiro F. Manuel d'EMDR (integration neuro-émotionnelle par les mouvements oculaires) principe, protocoles, procédures. Paris : Inter Éditions. 2007.

21. Shapiro F. Getting past your past. New York : Rodale Inc. 2012.
22. World Health Organization (2013). Guidelines for the management of Conditions Specifically Related to Stress. (2013). WHO Library Cataloguing-in-Publication Data. ISBN 9789241505406

23. Swift JK, Greenberg RP,. A Treatment by disorder meta-analysis of dropout from Psychotherapy. Journal of Psychotherapy Integration, 2014; Vol. 24, No. 3: 193-207.

24. Ho MSK, Lee CW. Cognitive behaviour therapy versus eye movement desensitization and reprocessing for posttraumatic disorder-is it all in the homework then? Revue Européenne de Psychologie Appliquée/ European Review of Applied Psychology. 2012; 62 (4): 253-260.

25. Marcus S, Marquis P, Sakai C. Three- and six - month follow up of EMDR treatment in an HMO setting. International Journal of Stress Management. 2004; 11: 195-208.

26. Schwabe L, Nader K, Pruessner JC. Reconsolidation of human memory : brain mechanisms and clinical relevance. Biol Psychiatry. 2014 [pii : S0006- 3223(14)00161-9. Epub ahead of print. Review].

27. Nader K, Hardt O, Lanius R. Memory as a new therapeutic target. Dialogues Clin Neurosci. 2013; 15(4): 475-86.

28. Wilson SA, Becker LA, Tinker RH. Eyemovement desensitization and reprocessing (EMDR) treatment for psychologically traumatized individuals. J Consult Clin Psychol. 1995; 63(6): 928-37.

29. Rothbaum BO. A controlled study of eye movement desensitization and reprocessing in the treatment of posttraumatic disordered sexual assault victims, Bulletin of the Menninger Clinic. 1997; 61(3): 317 334.

30. Wilson SA, Becker LA, Tinker RH. Fifteen-month follow-up of eye movement desensitization and reprocessing (EMDR) treatment of posttraumatic stress disorder and psychological trauma, Journal of Consulting and Clinical Psychology. 1997; 65(6): 1047-1056.

31. Marcus SV, Marquis P, Sakai C. Controlled study of treatment of PTSD using EMDR in an HMO setting. Psychotherapy. 1997; 34 (3): 307-315.

32. Marcus SV, Marquis P, Sakai C. Three and six months follow-up of EMDR treatment of PTSD in an HMO setting, International Journal of stress Management. 2004; 11(3): 195-208

33. De Jongh A, Ernst R, Marques L. The impact of eye movements and tones on disturbing memories involving PTSD and other mental disorders. J Behav Ther Exp Psychiatry. 2013; 44(4): 477-83 [Epub 2013 Jul 13].

34. van der Kolk BA, Spinazzola J, Blaustein ME, et al. A randomized clinical trial of EMDR, fluoxetine and pill placebo in the treatment of PTSD : treatment effect and long-term maintenance. J Clin Psychiatry. 2007; 68: 37-46.

35. Acarturk C, Konuk E, Cetinkaya M, et al. The efficacy of EMDR for posttraumatic stress disorder and depression among Syrian refugees : results of a randomized ontrolled trial. Psychol Med. 2016 Sep ; 46(12): 2583-93. doi : 10.1017/S0033291716001070. Epub 2016 Jun 29.

36. Schubert SJ, Le CW, de Araujo G, et al. The effectiveness of EMDR Therapy to treat symptoms following trauma in Timor Leste. J Trauma Stress. 2016 Apr; 29(2): 141-8. doi : 10.1002/jts.22084. Epub 2016 Mar 2.

37. Ter Heide FJ, Mooren TM, van de Schoot R, et al. EMDR Therapy v. stabilisation as usual for refugees : randomised controlled trial. Br J Psychiatry. 2016 Oct; 209(4): 311-318. Epub 2016 Feb 18.

38. Van Woudenberg C, Voorendonk EM, Bongaerts H, et al. Effectiveness of an intensive treatment programme combining prolongedexposure and EMDR for severe PTSD. European Journal of Psychotraumatology. 2018; VOL.9: 1487225

39. Yurtsever A, Konuk E, Akyuz T, et al. An EMDR group inntervention for Syrian refugees with PTSD : results of a randomized controlled trial. Front Psychol. 2018 June 12; 9: 463. 
40. Trentini C, Lauriola M, Giulani A, et al. Dealing with the Aftermath of Mass Disasters : A field study on the application of EMDR Integrative group treatment protocol with Child Survivors of the 2016 Italy earthqakes. Front Psychol. 2018 Jun 4; 9: 862.

41. Gillies D, Maiocchi L, Bhandari AP, et al. Psychological therapies for children and adolescents exposed to trauma. Cochrane Database Syst Rev. Cochrane Database Syst Rev. 2016 Oct 11; 10: CD012371.

42. Moreno- Alcazar A, Treen D, Valiente- Gomez A, et al. Efficacy of Eye Movement Desensitization and Reprocessing in Children and Adolescent with Post-traumatic Stress Disorder : A Meta-Analysis of Randomized Controlled Trials. Front Psychol. 2017 Oct 10; 8: 1750. doi : 10.3389/fpsyg.2017.01750. eCollection 2017.

43. de Roos C, van der Oord S, Zijlstra B, et al. Comparison of EMDR therapy, cognitive behavioral writing therapy, and wait- list in pediatric PTSD following single- incident trauma : a muliticenter randomized clinical trial. J Child Psychol Psychiatry. 2017 Nov; 58(11): 1219-1228. doi : 10.1111/jcpp.12768. Epub 2017 Jun 28.

44. Rodenburg R, Benjamin A, de Roos C, et al. (Efficacy of EMDR in children : a meta-analysis. Clin Psychol Rev. 2009; 29(7): 599-606 [Epub 2009 Jun 24].

45. Brennstuhl MJ, Tarquinio C, StrubL, et al. Benefits of immediate EMDR vs. eclectic therapy intervention for victims of physical violence and accidents at the workplace: a pilot study. Issues Ment Health Nurs. 2013;3 4(6): 425-34.

46. Tarquinio C, Rotonda C, Houllé WA, et al. Early psychological preventive intervention for workplace violence : A randomized controlled explorative and comparative study between EMDR- recent event and critical incident stress debriefing. Issues Ment Health Nurs. 2016 Nov; 37(11): 787-799. Epub 2016 Oct 3.

47. Gil-Jardiné C, Evrard G, Al Joboory S, et al. Emergency room intervention to prevent post concussion- like symptoms and PTSD. A pilot randomized controlled study of a brief EMDR intervention versus reassurance or usual care. J Psychiatr Res. 2018 Aug; 103: 229236. doi : 10.1016/j.jpsychires.2018.05.024. Epub 2018 May 26.

48. Van Etten M, Taylor LS. Comparative efficacy of treatments for post-traumatic stress disorder : a meta-analysis. Clin Psychol Psychotherapy. 1998; 5: 126-44.

49. [49] Bradley R, Greene J, Russ E, et al. A multidimensional metaanalysis of psychotherapy for PTSD. J Psychiatry. 2005; 162: 214-27.

50. Seidler G, Wagner F. Comparing the efficacy of EMDR and traumafocused cognitive behavioural therapy in the treatment of PTSD. A meta-analytic study. Psychol Med. 2006; 36: 1515-22.

51. Bisson JI, Ehlers A, Matthews R, et al. Psychological treatments for chronic post-traumatic stress disorder : systematic review and metaanalysis. Br J Psychiatry. 2007; 190: 97-104.

52. Lee CW, Cuijpers P. A meta-analysis of the contribution of eye movements in processing emotional memories. J Behav Ther Exp Psychiatry. 2013; 44: 231e239.

53. Haour F, de Beaurepaire C. Évaluation scientifique de la psychothérapie EMDR. Encephale. 2016 Jun; 42(3): 284-8. doi : 10.1016/j.encep.2016.02.012. Epub 2016 Mar 23. Review. French.

54. Valiente-Gómez A, Moreno-Alcázar A, Treen D, et al. EMDR beyond PTSD : A systematic Literature Review. Front Psychol. 2017 Sep 26; 8: 1668. doi : 10.3389/fpsyg.2017.01668. eCollection 2017.

55. Kutz I, Resnik V, Dekel R. The Effect of Single-Session Modified EMDR on Acute Stress Syndromes. Journal of EMDR Practice and Research. 2008; Volume 2, Number 3: 190-200(11).

56. Hase M, Balmaceda UM, Hase A, et al. EMDR therapy in the treatment of depression : a matched pairs in an inpatient setting. Brain Behav. 2015 Jun; 5(6): e00342. doi : 10.1002/brb3.342. Epub 2015 Apr 30.

57. A De Jongh, E Ten Broeke, MR Renssen. Treatment of specific phobias with EMDR : Protocol, empirical status and conceptual issues. Journal of Anxiety Disorders. 1999; 13 (1-2): 69-85.

58. A De Jongh, HJM Van den Oord, E Ten Broeke. Efficacy of EMDR in the treatment of specific phobias : four single- case studies on dental phobia. Journal of Clinical Psychology. 2002; 58 (12): 1489-1503.

59. De Jongh A, Holmshaw M, Carswell W, et al. "Usefulness of a traumafocused tratment approach for travel phobia". Clinical Psychology and Psychotherapy. 2011; 18: 124-137.

60. de Jongh A. Treatment of a woman with emetophobia : a trauma focused approach". Mental Illness. 2012 Jul 26; 4(1): e3. Published online 2012 Feb 3. doi : [10.4081/mi.2012.e3]

61. Markus W, de Weert-van Oene GH, Woud ML, et al. Are addiction - related memories malleable by working memory competition ? Transient effects on memory vividness and nicotine craving in a randomized lab experiment. J Behav Ther Exp Psychiatry. 2016 Sep; 52: 83-91. doi : 10.1016/j.jbtep.2016.03.007. Epub 2016 Mar 18.

62. Littel M, van den Hout MA, Engelhard IM. Desensitizing Addiction : Using EMDR ti reduce the intensity of substance- related mental imagery and craving. Front Psychiatry. 2016 Feb 8; 7: 14. doi : 10.3389/fpsyt.2016.00014. eCollection 2016.

63. Schäfer I, Chuey-Ferrer L, Hofmann A, et al. Effectiveness of EMDR in patients with substance use disorder and comorbid PTSD : study protocol for a randomized controlled trial. BMC Psychiatry. 2017 Mar 16; 17(1): 95. doi: 10.1186/s12888-017-1255-9.

64. C De Roos, AC Veenstra, A de Jongh, et al. Treatment of chronic phaom limb pain using a trauma- focused psychological approach. Pain Research and Management. 2010; 15 (2): 65-71.

65. Brennsthul MJ. Utilisation de la thérapie EMDR dans le cadre de la douleur chronique : efficience et perspectives cliniques. Thèse de troisième cycle, Université de Lorraine, document non publié. 2013.

66. Nazari H, Momeni N, Jariani M, et al. Comparison of eye movement desensitization and reprocessing with citalopram in treatment of obsessive compulsive disorder. International Journal of Psychiatry in Clinical Practice. 2011; vol.15 (4): 270-274.

67. Marsden Z, Lovell K, Blore D, et al. A randomized controlled trial comparing EMDR and CBT for obsessive- compulsive disorder. J Clin Psychol Psychother. 2018 Jan; 25(1): e10-e18. doi : 10.1002/ cpp.2120. Epub 2017 Jul 28.

68. Griffioen BT, van der Vegt AA, de Groot IW, et al. The effect of EMDR and CBT on low self-esteem in a genaral psychiatric population : a randomized controlled trial. Front Psychol. 2017 Nov 8; 8: 1910. doi : 10.3389/fpsyg.2017.01910. eCollection 2017.

69. Lobregt-van Buuren E, Sizoo B, Mevissen L, et al. EMDR therapy as a feasible and potentila effective treatment for adults with autism spectrum disorder (ASD) and a history of adverse events. J Autism Dev Disord. 2018 Jul 25. doi : 10.1007/s10803-018-3687-6. [Epub ahead of print]

70. De Bont PA, Van Minnen A, De Jongh A, et al. Patients with psychosis : a within-group controlled feasibility study examining the efficacy and safety of evidence-based PE and EMDR protocols. Behav Ther. 2013; 44(4): 717-30. [Epub 2013 Jul 27].

71. van den Berg DP, de Bont PA, van der Vleugel BM, et al. TraumaFocused treatment in PTSD patients With Psychosis : Symptom Exacerbation, Adverse Events, and Revictimization. Schizophr Bull. 2016 May; 42(3): 693-702. doi : 10.1093/schbul/sbv172. Epub 2015 Nov 24.

72. van den Berg DP, de Bont PA, van der Vleugel BM, et al. Prolonged exposure vs EMDR vs waiting list for posttraumatic stres disorder in patients with a psychotic disorder : a randomized clinical trial, JAMA Psychiatry. 2015 Mar; 72(3): 259-67. doi : 10.1001/ jamapsychiatry.2014.2637.

73. van Minnen A, van der Vleugel BM, van den Berg DP, et al. Effectiveness 
of trauma- focused treatment for patients with psychosis and without the dissociative subtype of post- traumatic stress disorder. Br J Psychiatry. 2016 Oct; 209(4): 347-348. Epub 2016 Aug 4.

74. de Bont PA, van den Berg DP, van der Vleugel BM, et al. Prolonged exposure and EMDR for PTSD v. a PTSD waiting list condition : effects on symptoms of psychosis, depression and social functioning in patients with chronic psychotic disorders. Psychol Med. 2016 Aug; 46(11): 2411-21. doi : 10.1017/S0033291716001094. Epub 2016 Jun 14 .

75. van den Berg D, de Bont PAJM, van der Vleugel BM, et al. Long term outcomes of trauma- focused treatment in psychosis. Br J Psychiatry. 2018 Mar; 212(3): 180-182. doi : 10.1192/bjp.2017.30. Epub 2018 Feb 7.

76. Haour F, Servan Schreiber D. Mécanismes neurobiologiques de l'EMDR. In : Cottraux J, editor. Les Thérapies Comportementales et Cognitives. Paris Masson. 2005.

77. Calancie OG, Khalid-Khan S, Booij L, et al. EMDR as a treatment for PTSD : current neurobiological theories and a new hypothesis. Ann N Y Acad Sci. 2018 Jun 21. doi : 10.1111/nyas.13882. [Epub ahead of print] Review ;

78. Wurtz H, El-Khoury-Malhame M, Wilhelm FH, et al. Preventing longlasting fear recovery using bilateral alternating sensory stimulation : a translational study, Neuroscience. 2016 May 3; 321: 222-235. doi : 10.1016/j.neuroscience.2015.06.012. Epub 2015 Jun 16.].

79. Servan-Schreiber D, Schooler J, Dew M et al. Eye movement desensitization and reprocessing for post-traumatic stress disorder : a pilot, blinded, randomized study of stimulation type. Psychother Psychosom. 2006; 75: 290-7.

80. Antrade J, Kavanagh D, Baddeley A. Eye movements and visual imagery : a working memory approach to the treatment of posttraumatic stress disorder. Br J Clin Psychol. 1997; 36: 209-23.

81. Engelhard IM, Van Uijen SL, Van den Hout MA. The impact of taxing working memory on negative and positive memories. Eur J Psychotraumatol. 2010; 1(5623): 1-8.

82. Van den Hout MA, Engelhard IM, Beetsm D, et al. EMDR and mindfulness : eye movements and attentional breathing tax working memory and reduce vividness and emotionality of aversive ideation. J Behav Ther Exp Psychiatry. 2011; 42: 423-31.

83. Nieuwenhuis S, ElzingaBM, Ras $\mathrm{PH}$, et al. Bilateral saccadic eye movements and tactile stimulation, but not auditory stimulation, enhance memory retrieval. Brain Cogn. 2013; 81(1): 52-6.

84. Pagani M, Amann BL, Landin-Romero R, et al. EMDR and slow wave sleep : a putative mechanism of action, Front Psychol. 2017 Nov 7; 8: 1935. doi : 10.3389/fpsyg.2017.01935. eCollection 2017.

85. Carletto S, Borsato T, Pagani. The role of slow wave sleep in memory pathophysiology : focus n PTSD and EMDR, M. Front Psychol. 2017 Nov 22; 8: 2050. doi : 10.3389/fpsyg.2017.02050. eCollection 2017. No abstract available.

86. Khalfa S, Elkhoury M, Guedj E, et al. Mécanismes cérébraux impliqués dans l'état de stress post-traumatique (ESPT) et son traitement par EMDR (Eye Movement Desensization and Reprocessing). Encéphale. 2012; 38(4S): 515

87. Pagani M, Di Lorenzo G, Verardo AR, et al. Neurobiological correlates of EMDR monitoring. An EEG study. PLoS One. 2012; 7(9): e45753.

88. Thomaes K, Engelhard IM, Sijbrandij M, et al. Degrading traumatic memories with EMDR : a pilot functional MRI study in PTSD. Eur J Psychotraumatol. 2016 Nov 29; 7: 31371. doi : 10.3402/ejpt. v7.31371. eCollection 2016

89. Boukezzi S, El Khoury-Malhame M, Auzias G, et al. Grey matter density changes of structures involved in PTSD after recovery following EMDR therapy. Psychiatry Res Neuroimaging. 2017 Aug 30; 266: 146-152. doi : 10.1016/j.pscychresns.2017.06.009. Epub 2017 Jun 19

90. Fleck JI, Olsen R, Tumminia M, et al. Changes in brain connectivity following exposure to bilateral eye movements. Brain Cogn. 2018 Jun; 123: 142-153. doi : 10.1016/j.bandc.2018.03.009. Epub 2018 Mar 21.

91. Laugharne J, Kullack C, Lee CW, et al. Amygdala volumetric change following psychotherapy for posttraumatic stress disorder. J Neuropsychiatry Clin Neurosci. 2016 Jun 3; appineuropsych16010006.

92. Khalaf O, Resch S, Dixsaut L, et al. Reactivation of recall- induced neurons contributes to remote fear memory attenuation. Science. 2018; 360: 1239-1242.

93. de Voogd LD, Kanen JW, Neville DA, et al. Eye-Movement Intervention Enhances Extinction via Amygdala Deactivation, Journal of Neuroscience. 3 October 2018; 38 (40): 8694-8706. DOI: https://doi. org/10.1523/JNEUROSCI.0703-18.2018

94. Denny NR. An orienting reflex/external inhibition model of EMDR and Thought Field Therapy. Traumatology. 1995; 1(1). http:// dx.doi.org/10.1177/153476569500100101

95. Stickgold R. EMDR: A putative neurobiological mechanism of action. Journal of Clinical Psychology. 2002; 58: 61-75.

96. Stickgold R. Sleep-dependent memory processing and EMDR action. Journal of EMDR Practice and Research. 2008; 2: 289-299. 